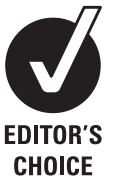

- Additional supplementary materials are published online only. To view these files please visit the journal online (http:// gut.bmj.com/content/61/8.toc).

${ }^{1}$ Sahlgrenska Center for Cardiovascular and Metabolic Research/Wallenberg Laboratory, Sahlgrenska University Hospital, Gothenburg, Sweden

${ }^{2}$ Department of Molecular and Clinical Medicine, University of Gothenburg, Gothenburg, Sweden

${ }^{3}$ Department of Microbiology, Cornell University, Ithaca, New York, USA

${ }^{4}$ Department of Chemical and Biological Engineering, Chalmers University of Technology, Gothenburg, Sweden

\section{Correspondence to}

Dr Fredrik Bäckhed, Wallenberg Laboratory, Sahlgrenska

University Hospital, S-413 45 Gothenburg, Sweden;

fredrik.backhed@wlab.gu.se

Revised 21 October 2011 Accepted 23 October 2011 Published Online First 23 November 2011

\section{UNLOCKA}

This paper is freely available online under the BMJ Journals unlocked scheme, see http:// gut.bmi.com/site/about/ unlocked.xhtml

\title{
Analysis of gut microbial regulation of host gene expression along the length of the gut and regulation of gut microbial ecology through MyD88
}

\author{
Erik Larsson, ${ }^{1,2}$ Valentina Tremaroli, ${ }^{1,2}$ Ying Shiuan Lee, ${ }^{1,2}$ Omry Koren, ${ }^{3}$ \\ Intawat Nookaew, ${ }^{4}$ Ashwana Fricker, ${ }^{3}$ Jens Nielsen, ${ }^{4}$ Ruth E Ley, ${ }^{3}$ Fredrik Bäckhed ${ }^{1,2}$
}

\section{ABSTRACT}

Background The gut microbiota has profound effects on host physiology but local host-microbial interactions in the gut are only poorly characterised and are likely to vary from the sparsely colonised duodenum to the densely colonised colon. Microorganisms are recognised by pattern recognition receptors such as Toll-like receptors, which signal through the adaptor molecule MyD88. Methods To identify host responses induced by gut microbiota along the length of the gut and whether these required MyD88, transcriptional profiles of duodenum, jejunum, ileum and colon were compared from germ-free and conventionally raised wild-type and Myd88-/- mice. The gut microbial ecology was assessed by 454-based pyrosequencing and viruses were analysed by PCR.

Results The gut microbiota modulated the expression of a large set of genes in the small intestine and fewer genes in the colon but surprisingly few microbiotaregulated genes required MyD88 signalling. However, MyD88 was essential for microbiota-induced colonic expression of the antimicrobial genes Reg3 $\beta$ and $\operatorname{Reg} 3 \gamma$ in the epithelium, and Myd88 deficiency was associated with both a shift in bacterial diversity and a greater proportion of segmented filamentous bacteria in the small intestine. In addition, conventionally raised Myd88-/mice had increased expression of antiviral genes in the colon, which correlated with norovirus infection in the colonic epithelium.

Conclusion This study provides a detailed description of tissue-specific host transcriptional responses to the normal gut microbiota along the length of the gut and demonstrates that the absence of MyD88 alters gut microbial ecology.

The human gut is home to trillions of bacteria (gut microbiota) that have co-evolved with us and established a finely tuned symbiosis. ${ }^{1}$ Their combined genomes (metagenome), which contain 150-fold more genes compared with our own genome, provide us with functions that we did not have to evolve ourselves. ${ }^{2}{ }^{3}$ Recent data suggest that if this symbiosis is disrupted we are exposed to the increased risk of developing common diseases such as inflammatory bowel disease and obesity. ${ }^{4}$ Germ-free mice provide a powerful tool to gain mechanistic insights into host-microbial interactions and their effect on host physiology. Colonisation of germ-free mice is associated with profound morphological changes in the small intestine such as shortening and widening of the

\section{Significance of this study}

What is already known about the subject?

- The gut harbours a vast ensemble of bacteria, the gut microbiota, encoding 150 -fold more genes than our own genome.

- The gut microbiota is altered in inflammatory bowel disease and obesity.

- Germ-free mice are protected against inflammatory bowel disease and diet-induced obesity.

- Toll-like receptors recognise microorganisms and signal via MyD88.

\section{What are the new findings?}

- Provides an extensive survey of host responses to the normal gut microbiota along the length of the gut.

- Analysis of the gut microbial ecology along the length of the gut.

- Demonstrates that Myd88-deficient mice harbour norovirus in the colonic epithelium.

\section{How might it impact on clinical practice in the} foreseeable future?

- Understanding the fundamental factors underlying host-microbial interactions in the mammalian gut is essential for future studies directed at targeting the gut microbiota in order to improve health. We also provide a webaccessible database, http://microbiota.wall.gu. se, to investigate whether specific genes are regulated by the gut microbiota and/or MyD88. This resource will facilitate the identification of microbially regulated genes for researchers interested in all aspects of gastroenterology.

villus, ${ }^{5}$ increased vascularisation, ${ }^{6}$ recruitment of lymphocytes ${ }^{7}$ and activation of the innate and adaptive immune systems. ${ }^{8}$

The innate immune system recognises bacteria and other infectious agents by pattern recognition receptors such as intracellular nucleotide-binding oligomerization domain-like receptors and Toll-like receptors (TLR). ${ }^{9}$ All TLR (except TLR3) as well as interleukin (IL) 1 and IL-18 signal via MyD88dependent pathways, which activate nuclear factor kappa B-driven pro-inflammatory signalling. Accordingly, MYD88 deficiency in humans is associated with increased susceptibility to pyogenic bacterial 
infections and Myd88-deficient mice have enhanced susceptibility and morbidity to most viral and bacterial experimental infections. ${ }^{10}$

The microbial ecology of the gut is governed by several factors, such as age, diet and host genotype, and recent findings suggest an important role for the innate immune system. ${ }^{11}$ In particular, TLR 5 has been shown to have a significant impact in shaping the colonic gut microbiota, whereas TLR2 and TLR4 appear to play a minor role. ${ }^{12}{ }^{13}$ Furthermore, in a mouse model of non-obese diabetes, Myd88 deficiency is associated with altered microbial community composition that confers protection against developing the disease. ${ }^{14}$

The function and architecture of the gut differs along its length: for example, nutrient absorption is most prevalent in the duodenum and jejunum, whereas the ileum is more immunologically active. ${ }^{15}$ The colon is more of a fermentative reactor producing short chain fatty acids and is the major site of water reabsorption. ${ }^{15}$ However, little is known about how the host responds to gut microbiota along the length of the gut. The concentration of bacteria increases along the length of the gut, from $10^{4}$ cells/g in the duodenum to $10^{12}$ cells/g in the colon, ${ }^{15}$ and most published studies of gut microbial ecology have focused on the colon and feces, with less emphasis on the less populated and more inaccessible small intestine. The aim of our study was to identify the influence of innate immunity on microbiota-induced host responses and microbial composition along the length of the gut; we used Myd88 deficiency as a model for the loss of innate immune signalling.

\section{MATERIALS AND METHODS \\ Mice}

Germ-free C57B16/J and Swiss Webster male mice (three to five per cage) were maintained in flexible film isolators under a $12-\mathrm{h}$ light cycle and freely fed an autoclaved chow diet (Labdiet, St Louis, Missouri, USA). The Myd88-/- were backcrossed at least eight generations to $\mathrm{C} 57 \mathrm{~B} 16 / \mathrm{J}$ and the last two crossings were performed using mice from our colony. These two lines were thereafter separated by a maximum of two generations. Germfree and conventionally raised mice were separated by a maximum of three generations. Conventionally raised mice were fed the same autoclaved diet upon weaning and all mice were used at 12 weeks of age.

Mice were killed by cervical dislocation and the small intestines, cecum and colon were removed. The small intestine was divided into eight equal-sized segments and the colon into three. For RNA analyses, we used the first (duodenum), fifth (jejunum), eighth (ileum) segments and the proximal piece of the colon. For analysis of the gut ecology, all segments were analysed. Epithelial cells were isolated as described previously. ${ }^{16}$ Animal protocols were approved by the Research Animal Ethics Committee in Gothenburg.

\section{RNA isolation}

RNA was isolated from the gut tissues and epithelial cells immediately after cell harvest using the RNeasy Mini Kit (Qiagen, Hilden, Germany). RNA concentration and quality were evaluated by spectrophotometric analysis (ND-1000; NanoDrop Technologies, Wilmington, DE, USA) and capillary electrophoresis on a 2100 Bioanalyzer (Agilent Technologies, Santa Clara, CA, USA).

\section{Microarray processing and statistical analysis}

RNA labelling, microarray hybridisation and scanning were performed at the Uppsala array platform core facility at Uppsala University using MoGene 1.0 ST chips (Affymetrix, Santa Clara, CA, USA), according to the manufacturer's instructions. Normalisation and probe set summarisation were performed on each tissue separately using the expression console software (Affymetrix). CEL files and normalised data were deposited into the NCBI GEO repository, accession number GSE17438. Downstream analyses were performed in Matlab using proprietary scripts as and functions from the Bioinformatics toolbox. Genes were annotated against the ENSEMBL ${ }^{17}$ set of genes using the MoGene 1.0 ST probe set mapping provided using BioMart. ${ }^{18}$ Probe sets that were mapped to more than one gene were excluded while redundant probe sets were averaged. This resulted in a final dataset of 22073 unique genes, each associated with one or more unambiguously mapped probe sets. Principal components analysis and hierarchical clustering (average linkage with Pearson's correlation coefficient) was performed on the processed dataset. Differential expression was evaluated in each tissue by calculating two-way analysis of variance (ANOVA) $p$ values, including a test for interaction, using colonisation status (germ-free or conventionally raised) and genotype (Myd88-/- or wild type) as independent variables. This test was applied to each individual gene, and was followed by adjustment for multiple comparisons using the $\mathrm{q}$ value method proposed by Benjamini and Hochberg. ${ }^{19}$ Gene ontology (GO) statistics, based on ENSEMBL annotated terms and their ancestors in the GO hierarchy, were calculated using Fisher's exact test. To account for multiple testing, we used permutation simulations to determine that an enrichment $p$ value threshold of $10^{-4}$ resulted in a low fraction of false positives $(<5 \%)$. Genes without annotated GO terms were excluded from the analysis.

\section{cDNA synthesis and quantitative PCR}

An aliquot of $0.5 \mu \mathrm{g}$ of total RNA was reverse transcribed (high capacity cDNA reverse transcription kit; Applied Biosystems, Foster City, California, USA) and SYBR green-based quantitative reverse transcription ( $\mathrm{qRT}$ )-PCR was performed as described previously. ${ }^{16}$ Primers are listed in supplementary table S1, available online only.

\section{Bacterial community composition analysis}

Intestines were removed, flash frozen in liquid nitrogen and divided into 12 segments. The small intestine was divided equally into eight segments, the ninth segment corresponded to the cecum, and the large intestine was sectioned into three equal pieces. Whole community DNA was isolated from the segments using the tissue and cells DNA isolation kit (MoBio, Carlsbad, CA, USA). DNA concentration and quality was evaluated by Quant-iT PicoGreen dsDNA assay kit (Invitrogen, Carlsbad, CA, USA) and a plate reader.

PCR amplification of $16 \mathrm{~S}$ ribosomal RNA genes was carried out using barcoded primers 27F-338R for the $\mathrm{V} 1-\mathrm{V} 3$ region of the $16 \mathrm{~S}$ rRNA gene. ${ }^{20}$ Three independent $50 \mu \mathrm{PCR}$ reactions were performed for each sample, each consisting of 2.5 U EasyA high fidelity enzyme, $1 \times$ buffer, $2 \mu \mathrm{M}$ of each primer and 10-100 ng of the DNA template. Reaction conditions consisted of one cycle of $2 \mathrm{~min}$ at $95^{\circ} \mathrm{C}, 30$ cycles of $30 \mathrm{~s}$ at $95^{\circ} \mathrm{C}, 45 \mathrm{~s}$ at $57^{\circ} \mathrm{C}, 60 \mathrm{~s}$ at $72^{\circ} \mathrm{C}$ and a final cycle of $2 \mathrm{~min}$ at $75^{\circ} \mathrm{C}$ on a thermocycler (Eppendorf, Hamburg, Germany). The PCR amplification products from each reaction were pooled and purified using Ampure magnetic purification beads (Agencourt Bioscience, Brea, CA, USA) and sequenced at the Cornell University Life Sciences Core Laboratories Centre (using Roche/454 FLX genome sequencer; Roche, Basel, Switzerland).

Sequences were analysed using the quantitative insights into microbial ecology (OIIME) software package. ${ }^{21}$ Sequences were removed from further analysis if their length was outside the range of 200-1000 nt, or if they contained ambigious bases, primer mismatches, homopolymer runs greater than six nucleotides, or 
uncorrectable barcodes. The remaining sequences were denoised ${ }^{22}$ and assigned to operational taxonomic units using UCLUST, ${ }^{23}$ with a $97 \%$ pairwise identity threshold, and classified taxonomically using Greengenes. ${ }^{24}$ Changes in bacterial abundance were compared using a two-tailed Student's t test with a Bonferroni correction for multiple comparisons. For tree-based analyses, single representative sequences for each operational taxonomic unit were aligned using PyNAST, and the phylogenetic tree used in the UniFrac $^{25}$ analysis was built using FastTree. ${ }^{26}$ Sequences were deposited into MG-RAST, accession number MGP151.
RESULTS AND DISCUSSION

Gene expression profiling of germ-free and conventionally raised wild-type and Myd88-/- mice

We performed 77 microarray hybridisations to obtain global transcriptional profiles from duodenum, jejunum, ileum and colon from germ-free and conventionally raised wild-type and Myd88-/- mice. Hierarchical clustering revealed distinct clusters representing each of the four tissues and further subclustering based on colonisation status and genotype (wild type or Myd88-/-; figure 1A). Clustering by genotype was most clearly
E CONV-R

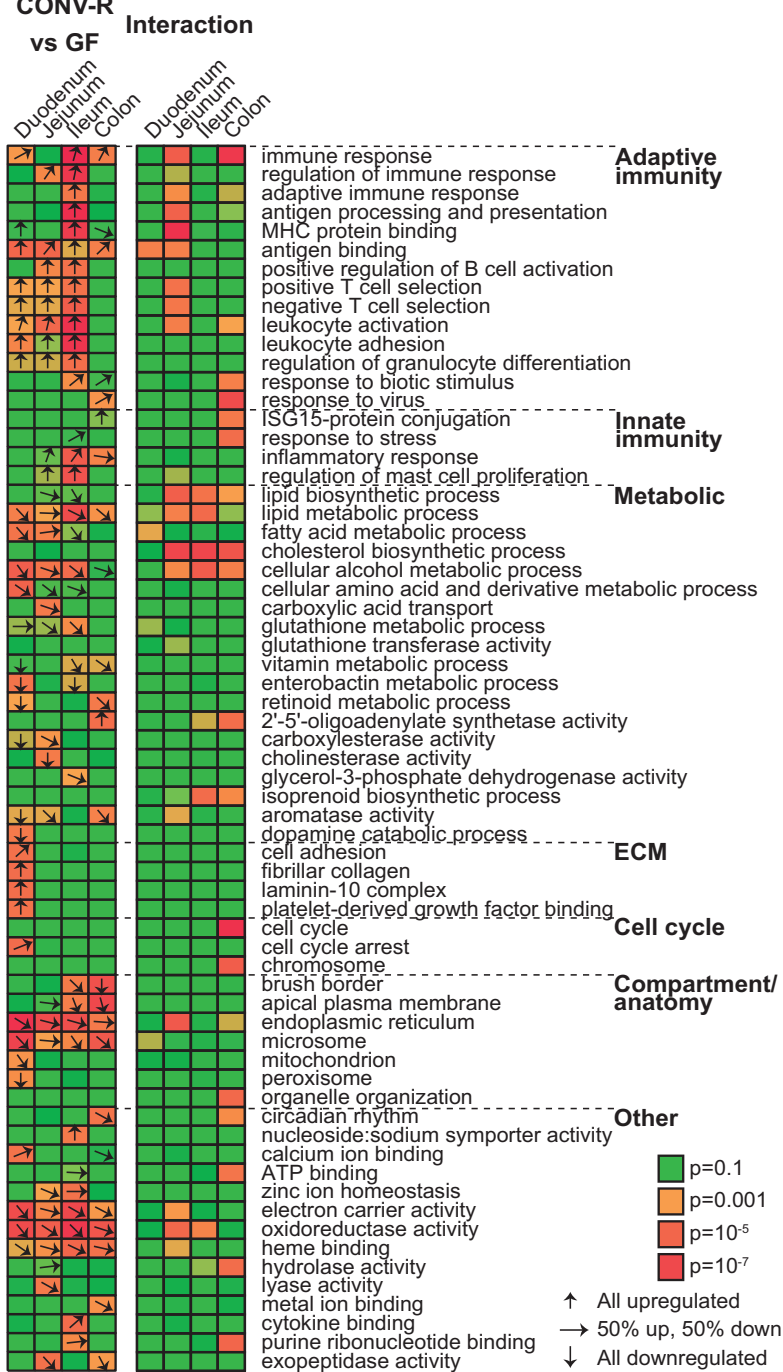

Figure 1 Transcriptional profiling of intestinal tissue samples from germ-free (GF) and conventionally raised (CONV-R) mice. (A) Hierachical clustering dendrogram of whole-transcriptome expression profiles obtained using DNA microarrays. (B) Principal component analysis of transcription profiles performed separately on each tissue. PC1, principal component 1; PC2, principal component 2. (C) All genes were evaluated by two-way analysis of variance for differential expression in germ-free versus conventionally raised mice (blue), Myd88-/- versus wild-type (green), as well as interaction between these two factors to reveal microbial responses that were modulated by Myd88 genotype (red). Quantile-quantile plots illustrate expected versus observed distributions of $p$ values obtained in these tests. Dotted lines show the expected distribution under the null hypothesis (no genes regulated). Strong deviations from this line indicate large effects on the transcriptome. (D) Venn diagram showing the number of differentially expressed genes (at $5 \%$ false discovery rate) between germ-free and conventionally raised mice in blue, $M y d 88-/-$ and wild-type mice in green, and genes that are regulated by the microbiota in a MyD88-dependent fashion (interaction) in red. (E) Enriched gene categories among regulated genes. The heat map indicates the level of statistical enrichment (Fisher's exact test) for select gene ontology (G0) categories among the 500 most significantly regulated genes. Representative $\mathrm{GO}$ terms were selected from the complete list of significant $\left(\mathrm{p}<10^{-4}\right.$, see Methods section) categories (see supplementary table $S 2$, available online only). The arrows indicate, in cases in which the enrichment is $p<0.01$, the proportion of genes (among top 500 ) in each category that are up or downregulated. Arrows are not shown for the interaction test as these per definition respond differentially depending on genotype. $n=4-6$ mice per group, but note that due to poor RNA quality of two duodenal samples from wild-type germ-free mice we only included two samples in the analyses. 
seen for colonised animals. A similar pattern was revealed by principal component analysis (figure $1 \mathrm{~B}$ ). We next applied a twoway ANOVA test to score for differential expression depending on colonisation status or genotype, as well as interaction between these factors to identify cases in which MyD88 modulates the microbial response. We observed dramatic transcriptional responses to the microbiota in all segments of the gut (figure 1C). In the small intestine, between 2844 and 5653 genes were regulated by the microbiota ( $5 \%$ false discovery rate; blue circles in figure 1D; a list of all microbiota-regulated genes is provided in supplementary table S2, available online only). Fewer genes were regulated by the microbiota in the colon compared with other segments (2124 genes; figure 1D). The limited microbial regulation in the colon may be explained by the presence of a thick continuous mucosal layer, which shields the epithelium from direct bacterial exposure. ${ }^{27}$

Given the importance of TLR as pattern recognition receptors for bacteria, we anticipated a major role for MyD88 in regulating the transcriptional responses to the gut microbiota. We observed a significant impact of MyD88 on host transcriptional profiles in the jejunum but not in other segments of the gut (figure 1D, green circle); however, these responses were independent of the gut microbiota (figure 1D, red circle). Although few microbiotainduced changes in gene expression required MyD88, such interaction effects became increasingly larger in the distal segments; 79 genes were significant in the interaction test in the colon at 5\% false discovery rate (red circles in figure 1D).

\section{Genes involved in immune responses and metabolism are predominantly regulated by the gut microbiota in the small intestine}

To identify major regulated pathways and processes, we evaluated the 500 most significant microbiota-regulated genes in each tissue for enrichment of functional gene categories. Several GO categories were statistically linked to microbial status in all tissues (figure 1E, see supplementary table S3, available online only). Similar to previous studies ${ }^{28-30}$ we observed significant microbial induction of genes related to immunity. The majority of these genes were associated with adaptive immunity, and are probably the result of lymphocyte migration to the mucosa and differentiation in response to the gut microbiota. ${ }^{7} 2831$ In addition, we evaluated transcriptional responses to the gut microbiota that depended on MyD88 (500 most significant genes in the interaction test). Immune categories were only weakly enriched in this set, although a stronger association was observed in the jejunum (figure 1E). Interestingly, these categories were also identified to be affected by MyD88 under germfree conditions in the jejunum and ileum.

Metabolism-related genes including lipid and fatty acid metabolism-related categories, as well as genes related to cellular compartments involved in nutrient absorption and metabolism, were regulated in response to the microbiota throughout the gut (figure 1E). Interestingly, genes related to cholesterol biosynthesis were enriched among the top genes that responded differentially to the microbiota depending on genotype in the jejunum, ileum and colon. Furthermore, genes associated with energy-yielding compartments, mitochondrion and peroxisome, were found to be among those downregulated in the duodenum of conventionally raised compared with germ-free mice.

\section{Most microbially regulated genes involved in immune responses and barrier function are not dependent on Myd88}

Expression levels of genes encoding immunoglobulins were altered in all segments of the gut, and this regulation was independent of MyD88 (see supplementary table S2, available online only). In agreement, we observed increased chemokine gene expression in gut tissues from conventionally raised compared with germ-free mice (table 1). By isolating primary epithelial cells from the ileum in a separate set of animals, we found that these genes were regulated by the gut microbiota in the epithelium (see supplementary figure S1A, available online only). An increase in chemokine levels would be expected to result in lymphocyte infiltration to the mucosa. ${ }^{7}$

We also observed microbiota-induced upregulation of genes associated with barrier function (table 1). Genes encoding small proline-rich protein $1 \mathrm{~A}$ and $1 \mathrm{~B}$, which play important roles as components of the cell envelope, and are cross-bridging proteins, $^{32}$ were significantly upregulated in the colon of conventionally raised mice (table 1). Expression of MUC2, the major mucin component of the colonic mucus layer, and expression of MUC4 and MUC13 were significantly elevated in all segments of the small intestine and colon (table 1). These responses were evident in epithelial cells (see supplementary figure S1B, available online only), but were unaffected by Myd88 deficiency (table 1).

\section{The inability to signal through MyD88 is associated with increased susceptibility to viral infections in conventionally raised mice}

Antiviral genes were induced by the microbiota in the colons of Myd88-/- mice but not in wild-type counterparts (figure 2). These results were corroborated by qRT-PCR (see supplementary figure S2, available online only) and could be localised to the epithelial layer (see supplementary figure S3, available online only). In contrast, we did not observe any increases in antiviral gene expression in the small intestine of $M y d 88-/-$ mice (see supplementary table S2, available online only).

At first these results may be counterintuitive as MyD88independent pathways are mainly invoked in the regulation of antiviral response genes. However, Myd88-deficient mice exhibit increased mortality in several models of experimental viral infections. ${ }^{10}$ To gauge whether the elevated expression of antiviral genes in the colonic epithelium of conventionally raised Myd88-deficient mice could be attributed to a viral infection, we performed a PCR-based screen of 15 selected viruses. This analysis revealed that our $M y d 88$-deficient mice were infected by murine norovirus (table 2). Murine norovirus is common in animal facilities around the world and does not cause symptoms in immune-competent mice. ${ }^{33}$

Our data thus suggest that Myd88-deficient mice are susceptible to naturally occurring viral infections and that the presence of norovirus in the colonic epithelium of $M y d 88$-deficient mice is the most likely cause of antiviral gene expression in these mice.

\section{Microbial regulation of REG3 $\beta$ and REG3 $\gamma$ requires MyD88 in colon but not ileum}

Two recently identified antimicrobial peptides, REG3 $\beta$ and REG3 $\gamma$, which are mainly produced by the Paneth cells but also by the absorptive epithelium, ${ }^{34}{ }^{35}$ were among the most significantly upregulated genes by the presence of a microbiota in both the small intestine and the colon (table 1). Interestingly, microbially induced expression of REG3 $\beta$ and REG3 $\gamma$ was dependent on MyD88 in the colon but not in the small intestine, (table 1), demonstrating distinct signalling pathways in these tissues. We also observed microbiota-induced expression of genes involved in the production of reactive oxygen (ROS) and nitrogen species (NOX1, NOS2 and DUOX2 and the maturation factor DUOXA2), which are also potent 


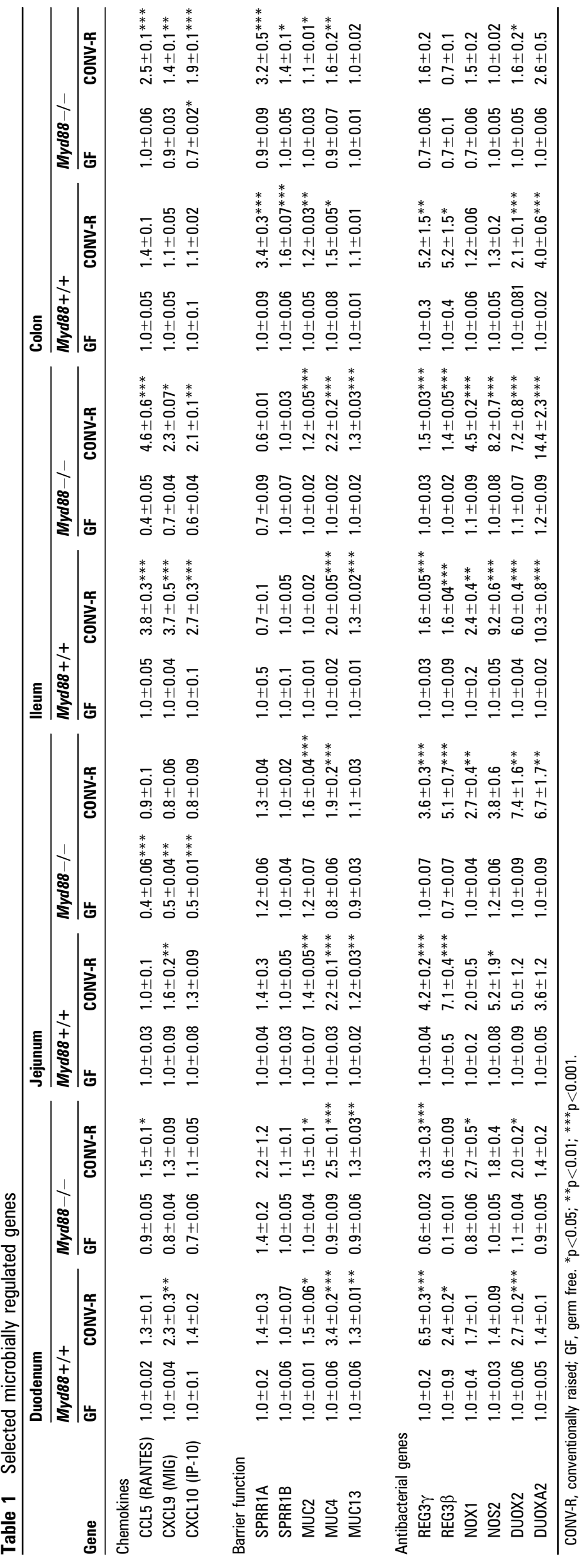


OAS2

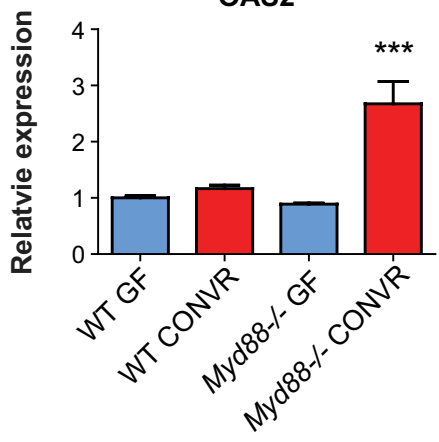

ISG15

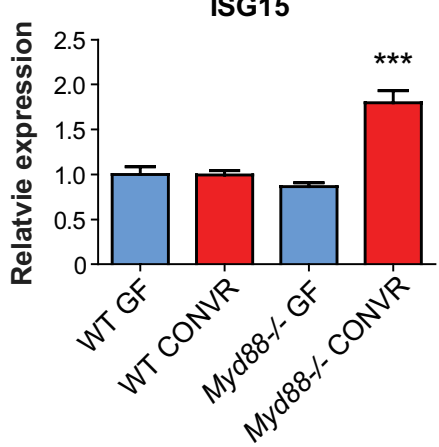

EIF2AK2

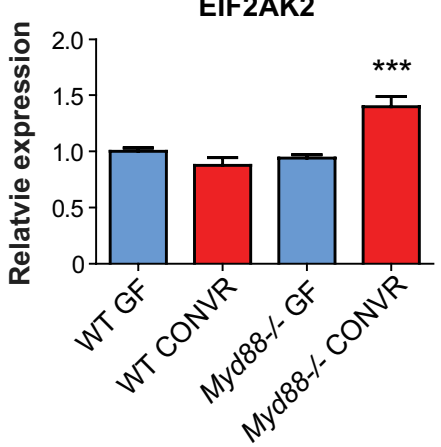

OAS1a

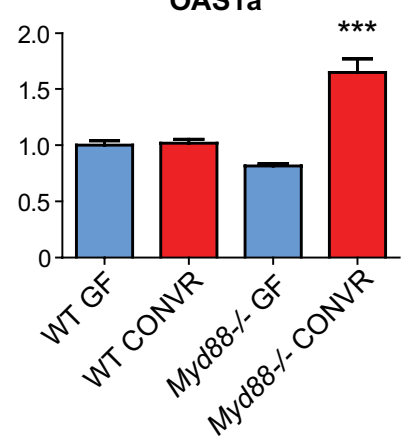

IFI2712A

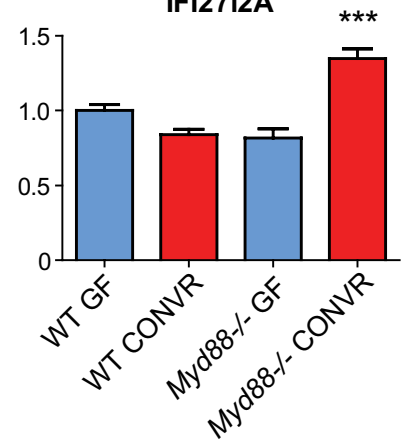

DDX58

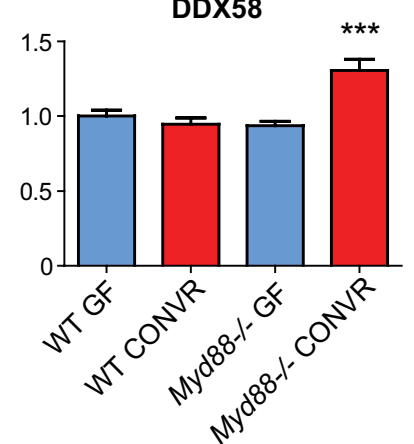

OAS1b

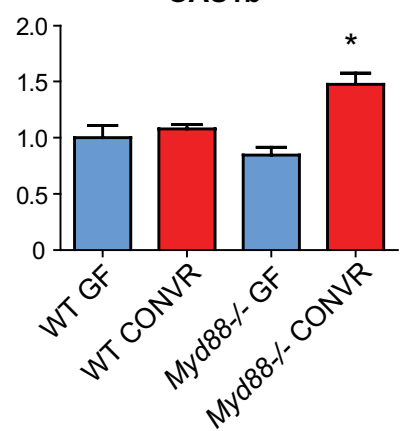

IRF7

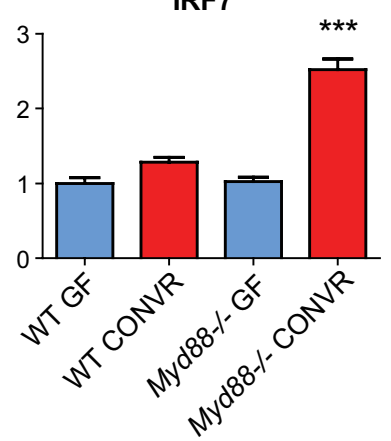

H2-Q8

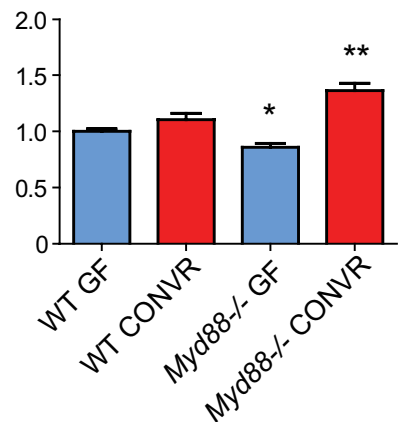

OASL2

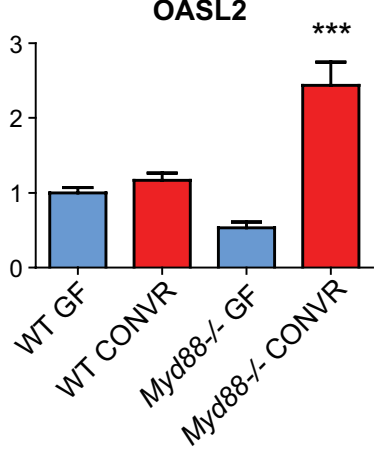

IFIH1

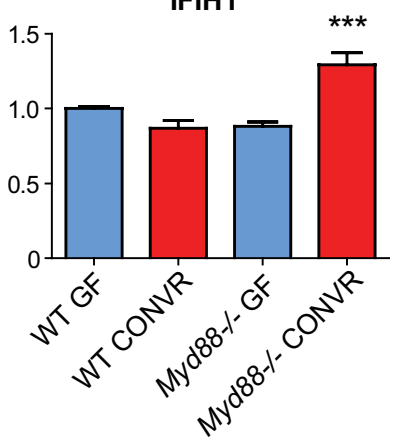

RSAD2

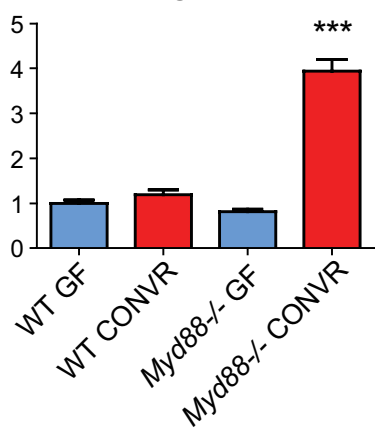

Figure 2 Relative expression levels of antiviral genes in the colon of germ-free (GF) and conventionally raised (CONV-R) wild-type (WT) and Myd88-/- mice. Data are from the microarray experiment and were corroborated by quantitated reverse transcription PCR in supplementary figure S2 available online only. $n=4-6$ per group. ${ }^{*} p<0.05,{ }^{* *} p<0.01,{ }^{* * *} p<0.001$; analysis of variance.

antimicrobial agents, ${ }^{36} 37$ with the most pronounced effect in the ileum (table 1; validated by qRT-PCR in supplementary table S4, available online only). However, in contrast to REG3 $\beta$ and REG3 $\gamma$, regulation of genes related to ROS production was independent of MyD88. qRT-PCR analysis of isolated epithelial cells from ilea and colons of germ-free and conventionally raised mice revealed that most of these genes were microbially regulated in the epithelium (see supplementary figure S4, available online only). Interestingly, we also noted a similar gene expression profile in Swiss Webster mice (see supplementary table S5, available online only), which are considered to have a $\mathrm{T}_{\mathrm{H}} 2$-biased immune system.

\section{MyD88 deficiency is associated with altered bacterial community composition}

We also examined bacterial diversity and community structure along the length of the gut in conventionally raised wild-type $(\mathrm{n}=7)$ and Myd88-deficient $(\mathrm{n}=4)$ mice using 454-based pyrosequencing of $16 \mathrm{~S}$ rRNA gene (see supplementary figure S5, available online only). Myd88-deficient mice had a significant increase in the relative abundance of segmented filamentous bacteria (SFB), especially in the jejunum and ileum (figure $3 \mathrm{~A}$ ), indicating that SFB are particularly affected by the loss of MyD88. SFB are tightly associated with the epithelium, especially in the small intestine, ${ }^{38}$ and play a fundamental role in the maturation of intestinal $\mathrm{T}$-cell responses and in the induction of gut inflammation, antimicrobial defence as well as mucosal IgA production. $^{28} 3940$ Therefore, it is possible to speculate that localisation of SFB close to the epithelium may make them more sensitive to antimicrobial molecules that are expressed by the epithelium in a MyD88-dependent fashion compared with other bacteria located further away from the surface of the epithelium.

To assess how the overall diversity of the microbiota was affected by Myd88 deficiency irrespective of the relative abundances of taxa, we compared samples using the unweighted UniFrac distance metric, which assumes that similar communities have similar evolutionary histories that can be represented as shared branches in a joint phylogenetic tree. ${ }^{25}$ This analysis revealed that the two genotypes harbour different bacterial communities (evidenced as clustering of samples by genotype in 
Table 2 PCR-based screening for mouse viruses in isolated colonic intestinal epithelial cells from conventionally raised mice

\begin{tabular}{lll}
\hline & Genotype & \\
\cline { 2 - 3 } Viral agent & Myd88+/+ & Myd88-/- \\
\hline Sendai virus & $-(0 / 3)$ & $-(0 / 3)$ \\
Mouse hepatitis virus & $-(0 / 3)$ & $-(0 / 3)$ \\
Pneumonia virus of mice & $-(0 / 3)$ & $-(0 / 3)$ \\
Minute virus of mice & $-(0 / 3)$ & $-(0 / 3)$ \\
Mouse parvovirus (MPV1, MPV2, MPV3) & $-(0 / 3)$ & $-(0 / 3)$ \\
Theiler's murine encephalomyelitis virus & $-(0 / 3)$ & $-(0 / 3)$ \\
Murine norovirus & $-(0 / 3)$ & $+(3 / 3)$ \\
Reovirus 3 & $-(0 / 3)$ & $-(0 / 3)$ \\
Mouse rotavirus (EDIM) & $-(0 / 3)$ & $-(0 / 3)$ \\
Ectromelia virus & $-(0 / 3)$ & $-(0 / 3)$ \\
Lymphocytic choriomeningitis virus & $-(0 / 3)$ & $-(0 / 3)$ \\
Polyoma virus & $-(0 / 3)$ & $-(0 / 3)$ \\
Lactate dehydrogenase-elevating virus & $-(0 / 3)$ & $-(0 / 3)$ \\
Mouse adenovirus (MAD1, MAD2) & $-(0 / 3)$ & $-(0 / 3)$ \\
Mouse cytomegalovirus & $-(0 / 3)$ & $-(0 / 3)$ \\
\hline
\end{tabular}

each of the different segments of the intestine, figure $3 \mathrm{~B}$ ). In addition, duodenum, jejunum and ileum-associated microbial communities show significantly higher levels of inter-mouse variation in bacterial diversity in Myd88-deficient mice compared with wild-type mice (see supplementary figure S6, available online only). In contrast, the bacterial communities in the cecum and colon clustered tightly (low mouse-mouse variation in microbial diversity, supplementary figure S6, available online only) and very clearly segregated by host genotype (figure 3B). Generally, difference in mouse to mouse variation along the length of the gut may reflect the biomass difference between gut segments. However, the greater mouse to mouse variation in Myd88-deficient animals compared with wild-type mice may signify a loss of control over microbial diversity.

Earlier studies have shown that the host genotype can affect the microbial ecology of the gut: for instance, $\alpha$-defensin deficiency has recently been shown to modify the composition of gut microbiota significantly in mice. ${ }^{41}$ We therefore propose that the MyD88-dependent increases in REG3 $\beta$ and REG3 $\gamma$ gene
Figure 3 Effect of host genotype on the microbiota composition along the length of the gut from conventionally raised wild-type and Myd88-/- mice. (A) Relative abundance of segmented filamentous bacteria (SFB) in the two genotypes (Myd88+/+ or $-/-$ ) along the length of the gut. ${ }^{* * *} \mathrm{p}<0.001$; two-tailed Student's t test with Bonferroni correction for multiple comparisons. (B) Principal coordinates analysis of unweighted UniFrac distances between bacterial communities determined from $16 \mathrm{~S}$ rRNA genes. Percentage variation explained by each principal coordinate $(P C)$ is indicated on the axis. Symbols repesent individual intestinal segments obtained from replicate mice: duodenum, first three segments; jejunum, segments $4-5$; ileum, segments 6-8; cecum, 9; large intestine, $10-12 . n=4-7$ mice per group. Low sequence counts were obtained for some samples, which were removed from further analysis.
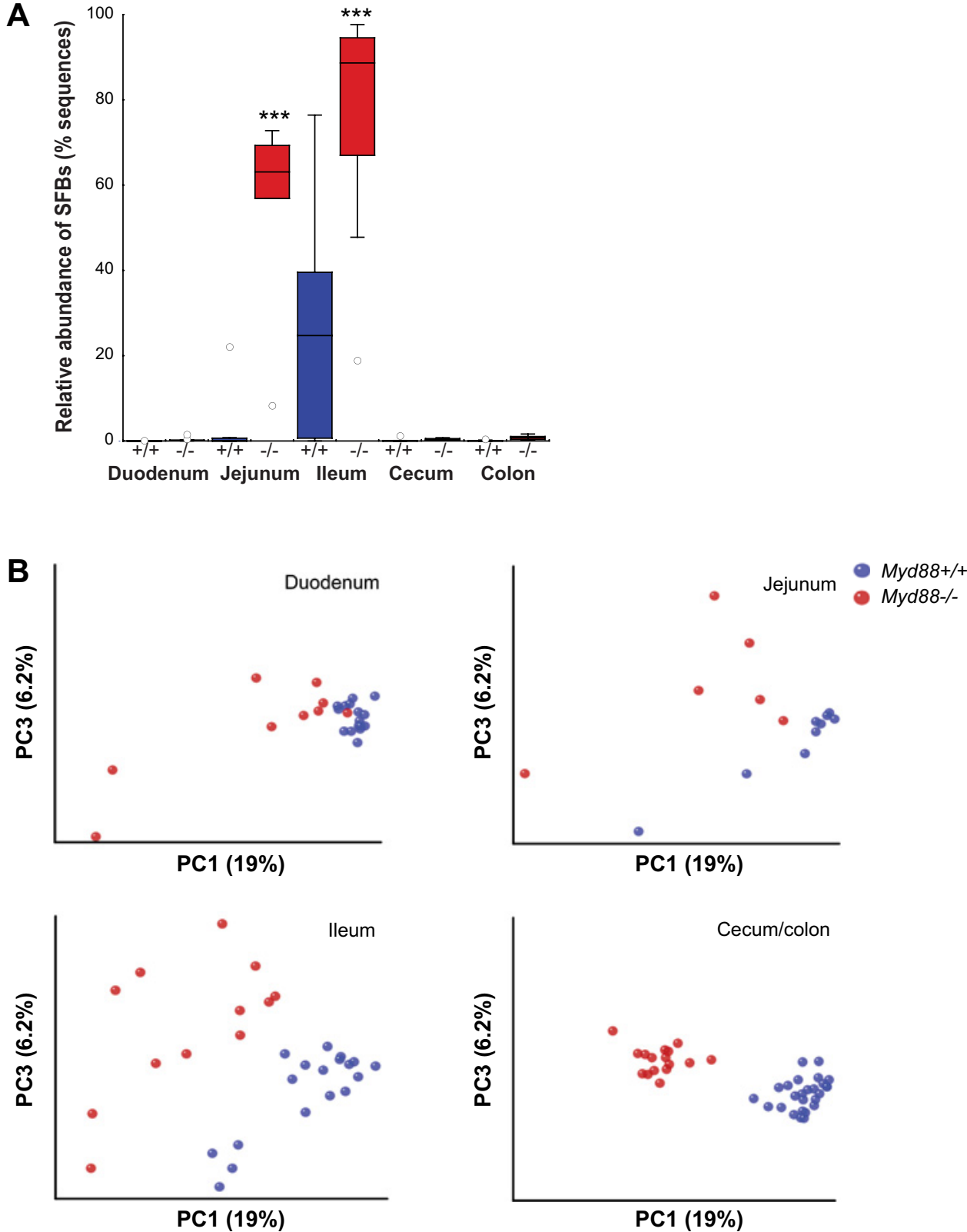
expression in the colon could contribute to the genotype-specific clustering of bacterial communities in the colon, but further experiments are required to prove this hypothesis.

\section{CONCLUSION AND PROSPECTUS}

In summary, we have extensively analysed the influence of the microbiota on gene expression and microbial ecology along the length of the gut, in both wild-type and Myd88-deficient animals. Furthermore, we identified limited but selective roles of MydD88 in increasing susceptibility to norovirus infections and modifying microbial ecology. Despite relatively small effects on mediating microbial-induced host gene expression changes, MyD88 affects host microbial ecology in the gut.

To make our gene expression data easily available to the scientific community, we constructed a searchable database (http://microbiota.wall.gu.se). The database enables interactive queries on gene name and detailed gene views with graphical illustrations of measured levels in different tissues and conditions, as well as statistical results and additional information about tissue expression profiles. $^{42}$ Data on host microbial responses in tissues (liver, epididymal and subcutaneous fat), that were not analysed in this report, are provided through the web interface.

Acknowledgements The authors would like to thank Carina Arvidsson, Anna Hallén and Gunnel Östergren-Lundén for superb technical assistance, Rosie Perkins for editing the manuscript, Aymé Spor for statistical support, and Jeffrey Gordon for supplying germ-free Myd88-/- mice.

Funding This work was supported by the Swedish Research Council (K2007-65X-20421-01-04), Swedish Foundation for Strategic Research, EU-funded ETHERPATHS projects (FP7-KBBE-222639, http://www.etherpaths.org) and TORNADO (FP7-KBBE-222720, http://www.fp7tornado.eu/), Åke Wiberg, Torsten and Ragnar Söderberg, Chalmers, Knut and Alice Wallenberg, and Novo Nordisk Foundations, and a LUA-ALF grant from Västra Götalandsregionen, as well as a Beckman young investigator award to REL.

Competing interests None

Contributors EL performed research, analysed data, wrote the manuscript; VT performed research, analysed data, commented on the manuscript; YL performed research, analysed data, commented on the manuscript; OK analysed data, commented on the manuscript; IN analysed data, commented on the manuscript; AF performed research; JN analysed data, commented on the manuscript; REL analysed data, assisting in writing the manuscript; FB conceived the study, analysed data, wrote the manuscript.

Provenance and peer review Not commissioned; externally peer reviewed.

Data sharing statement CEL files and normalised array data are available at NCBI GEO repository, accession number GSE17438. The expression data are also freely available through http://microbiota.wall.gu.se/. The 454 data set will be available at MGRAST—accession number MGP151.

\section{REFERENCES}

1. Ley RE, Hamady M, Lozupone C, et al. Evolution of mammals and their gut microbes. Science 2008;320:1647-51.

2. Oin J, Li R, Raes J, et al. A human gut microbial gene catalogue established by metagenomic sequencing. Nature 2010;464:59-65.

3. Turnbaugh PJ, Hamady M, Yatsunenko T, et al. A core gut microbiome in obese and lean twins. Nature 2009:457:480-4.

4. Ott SJ, Musfeldt M, Wenderoth DF, et al. Reduction in diversity of the colonic mucosa associated bacterial microflora in patients with active inflammatory bowe disease. Gut 2004;53:685-93.

5. Wostmann BS. The germfree animal in nutritional studies. Annu Rev Nutr 1981;1:257-79.

6. Stappenbeck TS, Hooper LV, Gordon Jl. Developmental regulation of intestinal angiogenesis by indigenous microbes via Paneth cells. Proc Natl Acad Sci U S A 2002:99:15451-5

7. Umesaki Y, Setoyama H, Matsumoto $S$, et al. Expansion of alpha beta T-cell receptor-bearing intestinal intraepithelial lymphocytes after microbial colonization in germ-free mice and its independence from thymus. Immunology 1993;79:32-7.
8. Slack E, Hapfelmeier S, Stecher B, et al. Innate and adaptive immunity cooperate flexibly to maintain host-microbiota mutualism. Science 2009;325:617-20.

9. Fukata M, Vamadevan AS, Abreu MT. Toll-like receptors (TLRs) and Nod-like receptors (NLRs) in inflammatory disorders. Semin Immunol 2009;21:242-53.

10. von Bernuth H, Picard C, Jin Z, et al. Pyogenic bacterial infections in humans with MyD88 deficiency. Science 2008;321:691-6.

11. Walter J, Ley RE. The human gut microbiome: ecology and recent evolutionary changes. Annu Rev Microbiol 2011:65:411-29.

12. Loh G, Brodziak F, Blaut M. The Toll-like receptors TLR2 and TLR4 do not affect the intestinal microbiota composition in mice. Environ Microbiol 2008;10:709-15.

13. Vijay-Kumar M, Aitken JD, Carvalho FA, et al. Metabolic syndrome and altered gut microbiota in mice lacking Toll-like receptor 5. Science 2010;328:228-31.

14. Wen L, Ley RE, Volchkov PY, et al. Innate immunity and intestinal microbiota in the development of type 1 diabetes. Nature 2008:455:1109-13.

15. Kleerebezem M, Vaughan EE. Probiotic and gut lactobacilli and bifidobacteria: molecular approaches to study diversity and activity. Annu Rev Microbiol 2009:63:269-90.

16. Reigstad CS, Lunden G0, Felin J, et al. Regulation of serum amyloid A3 (SAA3) in mouse colonic epithelium and adipose tissue by the intestinal microbiota. PLoS One 2009; 4:e5842.

17. Hubbard T, Barker D, Birney E, et al. The Ensembl genome database project. Nucleic Acids Res 2002;30:38-41.

18. Smedley D, Haider S, Ballester B, et al. BioMart - biological queries made easy. BMC Genomics 2009:10:22.

19. Benjamini Y, Hochberg $Y$. Controlling the false discovery rate: a practical and powerful approach to multiple testing. J Roy Statist Soc 1995;57:289-300.

20. Koren 0, Spor A, Felin J, et al. Human oral, gut, and plaque microbiota in patients with atherosclerosis. Proc Natl Acad Sci U S A 2010;108:4592-8.

21. Caporaso JG, Kuczynski J, Stombaugh J, et al. OlIME allows analysis of highthroughput community sequencing data. Nat Methods 2010;7:335-6.

22. Reeder J, Knight R. Rapidly denoising pyrosequencing amplicon reads by exploiting rank-abundance distributions. Nat Methods 2010;7:668-9.

23. Edgar RC. Search and clustering orders of magnitude faster than BLAST. Bioinformatics 2010;26:2460-1.

24. DeSantis TZ, Hugenholtz P, Larsen N, et al. Greengenes, a chimera-checked 16S rRNA gene database and workbench compatible with ARB. Appl Environ Microbiol 2006;72:5069-72.

25. Lozupone C, Knight R. UniFrac: a new phylogenetic method for comparing microbial communities. Appl Environ Microbiol 2005;71:8228-35.

26. Price MN, Dehal PS, Arkin AP. FastTree: computing large minimum evolution trees with profiles instead of a distance matrix. Mol Biol Evol 2009:26:1641-50.

27. Johansson ME, Phillipson M, Petersson J, et al. The inner of the two Muc2 mucindependent mucus layers in colon is devoid of bacteria. Proc Natl Acad Sci U S A 2008:105:15064-9.

28. Gaboriau-Routhiau V, Rakotobe S, Lecuyer E, et al. The key role of segmented filamentous bacteria in the coordinated maturation of gut helper $T$ cell responses. Immunity 2009;31:677-89.

29. Hooper LV, Wong MH, Thelin A, et al. Molecular analysis of commensal hostmicrobial relationships in the intestine. Science 2001:291:881-4.

30. Rawls JF, Mahowald MA, Ley RE, et al. Reciprocal gut microbiota transplants from zebrafish and mice to germ-free recipients reveal host habitat selection. Cell 2006;127:423-33.

31. Macpherson AJ, Hunziker L, McCoy K, et al. IgA responses in the intestinal mucosa against pathogenic and non-pathogenic microorganisms. Microbes Infect 2001:3:1021-35

32. Steinert PM, Marekov LN. Initiation of assembly of the cell envelope barrier structure of stratified squamous epithelia. Mol Biol Cell 1999;10:4247-61.

33. Pritchett-Corning KR, Cosentino J, Clifford CB. Contemporary prevalence of infectious agents in laboratory mice and rats. Lab Anim 2009;43:165-73

34. Mukherjee S, Partch CL, Lehotzky RE, et al. Regulation of C-type lectin antimicrobial activity by a flexible N-terminal prosegment. J Biol Chem 2009;284:4881-8.

35. Cash HL, Whitham CV, Behrendt CL, et al. Symbiotic bacteria direct expression of an intestinal bactericidal lectin. Science 2006;313:1126-30.

36. Nathan C. Inducible nitric oxide synthase: what difference does it make? J Clin Invest 1997; 100:2417-23.

37. Leto TL, Geiszt M. Role of Nox family NADPH oxidases in host defense. Antioxid Redox Signal 2006;8:1549-61.

38. Davis CP, Savage DC. Habitat, succession, attachment, and morphology of segmented, filamentous microbes indigenous to the murine gastrointestinal tract. Infect Immun 1974;10:948-56.

39. Klaasen HL, Van der Heiiden PJ, Stok W, et al. Apathogenic, intestinal, segmented filamentous bacteria stimulate the mucosal immune system of mice. Infect Immun 1993:61:303-6.

40. Ivanov II, Atarashi K, Manel N, et al. Induction of intestinal Th17 cells by segmented filamentous bacteria. Cell 2009;139:485-98.

41. Salzman NH, Hung K, Haribhai D, et al. Enteric defensins are essential regulators of intestinal microbial ecology. Nat Immunol 2010;11:76-83.

42. Su Al, Wiltshire T, Batalov $S$, et al. A gene atlas of the mouse and human protein-encoding transcriptomes. Proc Natl Acad Sci U S A 2004:101:6062-7. 